Musées, Patrimoine et Culture scientifiques et techniques

$160 \mid 2015$

juillet-août 2015

\title{
Le musée, le lieu de toutes les rencontres
}

\section{Éric Ferron}

\section{OpenEdition}

Journals

Édition électronique

URL : http://journals.openedition.org/ocim/1552

DOI : 10.4000/ocim.1552

ISSN : 2108-646X

\section{Éditeur}

OCIM

Édition imprimée

Date de publication : 1 juillet 2015

Pagination : 28-30

ISSN : 0994-1908

Référence électronique

Éric Ferron, «Le musée, le lieu de toutes les rencontres », La Lettre de l'OCIM [En ligne], 160 | 2015, mis en ligne le 01 juillet 2016, consulté le 02 mai 2019. URL : http://journals.openedition.org/ocim/1552 ; DOI : 10.4000/ocim.1552

Ce document a été généré automatiquement le 2 mai 2019.

Tous droits réservés 


\title{
Le musée, le lieu de toutes les rencontres
}

\author{
Éric Ferron
}

L'article (disponible sur www.ocim.fr, rubrique La Lettre de l'OCIM) décrivait en particulier l'expérimentation de nouveaux modes de visites et de mises en espace.

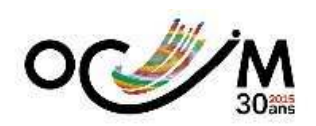

Pour voir les musées autrement...

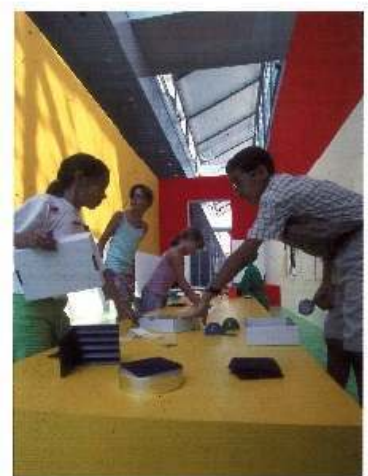

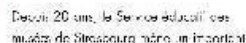

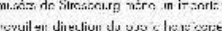

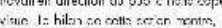

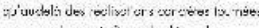

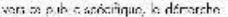

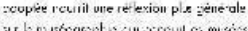

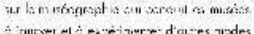

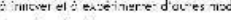

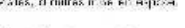

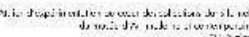

loction éducotive paur les grcupes
J'enlants ot de ieviles

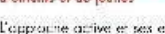

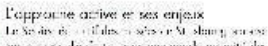
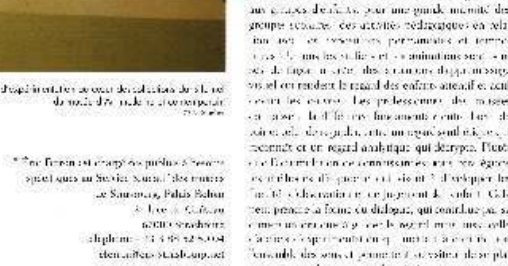

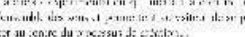

1 En 2003, il y a maintenant plus de dix ans, j'écrivais dans La Lettre de l'OCIM un article intitulé Pour voir les musées autrement... qui présentait la manière dont le service des publics des musées de Strasbourg envisageait la médiation à destination du public en 
situation de handicap. J'y présentais des visites, des objets muséographiques et des outils d'aide à la visite adaptés qui découlaient de la réflexion et des expériences menées depuis les années 1970 par Margaret Pfenninger, en charge du service des publics.

Le public en situation de handicap a toujours occupé une place à part dans la réflexion du service des publics, non pas parce qu'il est un public différent mais bien parce qu'il est un public qui oblige à nous interroger sur les notions premières et fondamentales d'accueil, de rapport à l'espace et de rencontre entre un individu et une œuvre d'art ou un objet ethnologique. Plutôt que de se référer à un visiteur moyen, virtuel, souvent fantasmé qui mène au concept de grand public et qui conduit à délivrer des messages réducteurs ou spectaculaires, il est beaucoup plus pertinent et respectueux, tant vis-à-vis de l'œuvre que des visiteurs, de se référer au public en situation de handicap. Ce dernier amène à penser et à élaborer des contextes de visite qui proposent à chaque visiteur de rencontrer véritablement l'œuvre sans occulter ce qu'il est en tant qu'individualité, avec son histoire, ses connaissances, sa sensibilité. C'est pourquoi, dix années après, il me semble que les approches présentées dans ce numéro de mars 2003 sont toujours justes.

\section{Faire du musée le lieu de toutes les rencontres}

Penser l'accueil du visiteur en situation de handicap et sa rencontre avec une œuvre ou un objet oblige à créer des situations de visite qui l'amènent à sentir et ressentir pour qu'il puisse ensuite y penser. Cette exigence fait directement écho à l'objectif premier de la médiation dans les musées qui consiste à abolir la distance entre le visiteur et l'œuvre ou l'objet et de mettre en relation l'anima, au sens du souffle, de l'âme qui émane intrinsèquement de toute œuvre, de tout objet avec celle de chaque visiteur. Il n'est donc ici jamais besoin d'animer ce qui l'est déjà mais de dénouer le hiatus de l'exposition et du temps qui éloignent l'objet de la vie en générale et de celle du visiteur en particulier.

Chaque visiteur et cela peu importe son handicap, son âge, sa sensibilité ou sa connaissance a besoin de se relier de manière sensible à une œuvre ou un objet pour véritablement la/le rencontrer. Plus que l'élève ou l'apprenant, c'est bien à l'individu auquel il s'agit de s'adresser pour qu'il se confronte à l'œuvre d'une manière personnelle et individuelle. Plutôt que d'animer une visite en imposant un discours ou un regard, il s'agit de créer les conditions pour que chaque visiteur qu'il soit venu seul, en famille ou avec des amis éprouve un objet, une œuvre et entre en dialogue avec lui. Pour y arriver, il existe différents moyens qui vont de l'accueil par la mise à disposition d'un vestiaire ou d'un siège pour créer des situations de confort, l'accompagnement dans les collections par la conduite de visites ou la mise à disposition d'outils adaptés en passant par les ateliers de pratique, autant d'éléments qui doivent avant tout poursuivre l'objectif de convoquer les sens, les émotions, la mémoire, la créativité ou la spiritualité parce que contempler une œuvre est avant tout une expérience de vie. La rencontre peut, comme j'ai pu le présenter et le décrire dans l'article de 2003, prendre la forme du dialogue, de l'échange, de la manipulation, du jeu ou encore de l'expérimentation du processus créatif.

Pour mettre en place ce type de situation de visite, il faut de la part du médiateur une bonne connaissance de l'œuvre ou de l'objet, s'appuyant sur des recherches et notamment celles du conservateur. Le couple conservateur-médiateur est primordial car il s'agit toujours de proposer de poser un regard pertinent sur l'œuvre. Il n'existe ainsi 
pas d'exposition exigeante qui s'imposerait par la seule excellence de son propos scientifique sans réflexion sur les conditions de visite et la rencontre sensible des œuvres.

\section{Le musée un lieu de transformation}

6 L'approche du visiteur face à l'œuvre d'art ou l'objet ethnologique s'articule donc entre deux processus : l'appel aux sens, c'est-à-dire voir, entendre, toucher, sentir et l'attention délibérée qui mène à la réflexion et à la connaissance. Une visite au musée qui n'inviterait pas à l'appel des sens, qui tendrait au contraire à contraindre les corps et à inhiber les sens imposerait au visiteur de rencontrer les œuvres par ses seules connaissances en histoire de l'art, en histoire, en esthétique, en théologie, en géographie ou en ethnologie. Le musée tendrait ainsi à écarter le public en situation de handicap mais aussi les enfants qui ont besoin d'apprendre par l'expérience, les familles ou encore ceux qui estiment ne pas disposer des références ou des connaissances nécessaires ou qui ne sont pas en mesure de déconstruire ces codes et de les dépasser. La violence sociale symbolique, concept développé par le sociologue Pierre Bourdieu, est d'ailleurs à mettre en relation avec les résultats des nombreuses enquêtes sur les publics montrant qu'il est constitué toujours des mêmes catégories socioprofessionnelles. Cela ne veut pas dire que le musée ne participe pas à faire fonctionner l'ascenseur social à travers notamment son rôle joué aux côtés de l'Éducation nationale mais qu'il attire dans ses collections en visiteurs libres toujours le même profil de personnes. Pourtant, si un visiteur dispose de connaissances qui lui permettent de se mettre en contact avec une œuvre sur le plan culturel, elles ne constituent pas le gage qu'il aura ensuite la ressource sensible suffisante pour pénétrer l'armure de l'œuvre et d'en sentir le souffle. Comprendre ce que l'on ressent appartient bien au domaine de la sensation et relève de la subjectivité, rester au simple stade de la connaissance revient à ce que l'art se retranche de la vie, prenne son indépendance et qu'il se suffise à lui-même. Le musée est ici visité comme un temple de la connaissance, un lieu où l'on se distingue, où l'on se reconnaît.

7 Pour le philosophe Emmanuel Levinas, il n'y a de rencontre qu'avec un autre, c'est-à-dire avec le dissemblable. Ainsi, si le visiteur regarde l'œuvre, la sent pour l'éprouver et la vivre comme participant de son identité, s'il accepte de se perdre dans ses certitudes et de l'accueillir avec toute sa valeur pour l'intégrer à sa réflexion, alors il la rencontrera et à travers elle, il rencontrera l'autre: cet artiste ou cette personne venue d'une autre époque, d'un autre territoire, d'un ailleurs. Le musée est bien un temple mais en tant que lieu de conservation des œuvres et des objets, un lieu qui doit autant proposer un propos scientifique rigoureux, apporter des références et de la connaissance que questionner la société, entrer en résonnance avec l'identité du visiteur qui s'est par ailleurs construite à partir de séries d'identifications à des références et des référents, d'expériences vécues et de sensations éprouvées. C'est en cela que le musée doit être pensé comme un lieu d'épanouissement et de transformation des individus et que le temps de la visite est bien le moment de créer l'authenticité et l'intimité de la rencontre.

\section{Le rôle social du musée}

8 En mettant l'œuvre sur un piédestal et accessible par la seule connaissance, le musée l'éloigne de la vie et le retranche de la sphère sociale. Pourtant si le musée a en France une mission de service public, c'est bien parce qu'il a un rôle de conservation mais aussi 
un rôle social en matière d'éducation, d'inclusion et de cohésion sociale. Pour y contribuer, le médiateur doit travailler de manière concertée et complémentaire avec les autres acteurs du territoire pour que la venue au musée ne se cantonne pas à une sortie mais constitue bien une entrée dans la vie notamment des personnes qui en sont ou s'en sont éloignées.

Dans Les Dossiers de l'OCIM publiés en 2011 et qui s'intitulaient Le rôle social du musée. Agir ensemble et créer des solidarités et qui se demandait comment les musées peuvent-ils répondre à cette exigence?, je présentais comment en Alsace, les structures culturelles, sociales et médico-sociales se sont réunies dans un réseau par le biais d'une association du nom de Tôt ou t'Art pour participer à l'inclusion sociale des individus et la cohésion sociale des territoires.

10 L'association Tôt ou t'Art anime un réseau à dimension régionale constituée aujourd'hui de près de 100 structures culturelles dont de nombreux musées et de plus de 200 structures sociales et médico-sociales dont $25 \%$ appartiennent au domaine du handicap. Elle anime un site Internet collaboratif qui met en lien plus de 2000 professionnels et qui facilite la venue du public en situation de handicap ou en difficulté sociale en communiquant sur la programmation et l'action culturelles adaptées, en proposant des facilités de billetterie, une tarification unique ou en proposant des formations, des actions de sensibilisation ou encore des temps d'échange de pratique.

11 L'association qui compte deux salariés, dispose d'un budget alimenté par la DRAC, l'Agence nationale pour la cohésion sociale et l'égalité des chances (ACsé), les collectivités territoriales et notamment la Ville et l'Eurométropole de Strasbourg, le Conseil départemental du Bas-Rhin, la Région Alsace mais également par les structures culturelles et sociales qui acquittent une cotisation au titre de leur adhésion. L'un des premiers objectifs de l'association est de rendre visible auprès des passeurs de culture que sont les éducateurs, les assistants sociaux, les infirmiers, les psychologues ou encore les conseillers en économie sociale et familiale, la programmation et les actions culturelles adaptées. Ceux-ci s'en font les relais auprès des personnes qu'ils accompagnent, qu'elles soient en situation de handicap ou en difficultés sociales ou encore vers leurs parents et organisent des visites qui s'inscrivent souvent dans un cadre plus large. Un autre objectif est de permettre aux professionnels de la culture et du social de s'approprier leurs champs d'intervention respectifs, les missions de chacun, pour ensuite mener des projets communs qui fassent sens pour des personnes qui sont le plus souvent isolées, coupées de leurs sensations et en recherche d'identité.

12 En 2011, en organisant pendant une année des tables rondes qui ont réuni plus de 120 professionnels, l'association a rédigé une charte d'accueil des publics du champ social dans les institutions culturelles qui avait pour ambition de créer sur le territoire les conditions structurelles de la venue de ces publics. Cette charte prévoyait notamment la création d'un référent dans chaque structure culturelle mais aussi sociale et médicosociale en charge de coordonner la venue de ce public dans les lieux culturels. C'est par ailleurs cette charte qui a permis de réaliser le cahier des charges du site collaboratif de l'association, sorte de réseau social où chaque entité dispose d'une page pour présenter qui son public, qui sa programmation et ses actions culturelles. En 2015, le site ira plus loin dans cette volonté de mise en lien en se transformant en un site ressource répertoriant toutes les expériences de visites et de muséographies adaptées. 
Lorsqu'en 2011, j'ai quitté la Direction de la Culture pour la Direction des Solidarités et de la santé de l'Eurométropole de Strasbourg, cela participait de cette volonté d'aller de l'autre côté du miroir et de vivre une expérience pleine et entière avec des publics souvent éloignés des musées ainsi qu'avec les professionnels qui les accompagnent. Devenu par ailleurs président de l'association Tôt ou t'Art à titre bénévole en 2009, je travaille toujours à faire du musée un lieu d'épanouissement et de transformation des individus en facilitant la venue des personnes en situation de handicap ou en difficulté sociale qui sont les visiteurs qui questionnent le plus les collections et l'institution et à faire en sorte que cette vision du musée soit l'affaire de tous, médiateurs mais aussi conservateurs, professionnels de la santé et du social et pouvoirs publics.

\section{RÉSUMÉS}

À l'occasion des 30 ans de l'OCIM, La Lettre de l'OCIM propose à ses lecteurs, dans chaque numéro de l'année 2015, un retour sur un article marquant de l'histoire de la revue. À partir d'un corpus d'articles choisis par la rédaction de La Lettre de l'OCIM, les membres du comité des Publications de l'OCIM ont sélectionné plusieurs contributions.

Dans cette perspective, il a été demandé à l'auteur ou à un expert du domaine de revisiter la problématique exposée dans l'article à la lueur des changements intervenus - notamment dans les pratiques professionnelles - depuis son écriture et de proposer des éléments prospectifs sur la question.

Éric Ferron effectue un retour sur son article "Pour voir les musées autrement..." publié en novembre-décembre 2003 dans le $n^{\circ} 90$ de La Lettre de l'OCIM et dans lequel, dressant le bilan du travail mené en direction du public handicapé par les musées de Strasbourg, il montrait comment cette démarche avait conduit les musées à innover.

\section{INDEX}

Mots-clés : musée, handicap

\section{AUTEUR}

\section{ÉRIC FERRON}

responsable des structures d'accueil et d'hébergement d'urgence à la Ville et Eurométropole de Strasbourg

eric.ferron@strasbourg.eu 